

\begin{tabular}{lcc}
\hline Neuron Parameters & & \\
\hline Spike threshold & $u_{t h}$ & $20 \mathrm{mV}$ \\
Reset potential & $u_{r}$ & $14 \mathrm{mV}$ \\
Resting potential & $u_{r e s t}$ & $0 \mathrm{mV}$ \\
Refractory period & $t_{r e f}$ & $1 \mathrm{~ms}$ \\
Membrane capacitance & $C_{m}$ & $250 \mathrm{pF}$ \\
Membrane time constant & $\tau_{m}$ & $10 \mathrm{~ms}$ \\
\hline Synaptic Parameters & & \\
\hline Synaptic rise time & $\tau_{r}$ & $1 \mathrm{~ms}$ \\
Synaptic decay time & $\tau_{d}$ & $1 \mathrm{~ms}$ \\
Synaptic weight & $J$ & \\
Synaptic delay & $d$ & $2 \mathrm{~ms}$ \\
\hline Input Parameters & & \\
\hline Inhibitory network & & $14 \mathrm{mV}$ \\
Input, mean - I population & $\mu_{I}$ & $6 \mathrm{mV}$ \\
Input, variance - I population & $\sigma_{I}$ & \\
& & \\
Excitatory-Inhibitory network & & $20.8 \mathrm{mV}$ \\
Input, mean - E population & $\mu_{E}$ & $16.5 \mathrm{mV}$ \\
Input, mean - I population & $\mu_{I}$ & \\
Input, variance - E population & $\sigma_{E}$ & $20.8 \mathrm{mV}$ \\
Input, variance - I population & $\sigma_{I}$ & $16.5 \mathrm{mV}$ \\
& &
\end{tabular}

\begin{tabular}{|c|c|c|}
\hline \multicolumn{3}{|l|}{ Network Parameters } \\
\hline \multicolumn{3}{|l|}{ Inhibitory network } \\
\hline Number of inhibitory neurons & $N_{I}$ & 10,000 \\
\hline \multicolumn{3}{|l|}{ Excitatory-Inhibitory network } \\
\hline Number of excitatory neurons & $N_{E}$ & 8,000 \\
\hline Number of inhibitory neurons & $N_{I}$ & 2,000 \\
\hline
\end{tabular}

\section{Control Parameters}

Inhibitory network

Control input

$\begin{array}{cc}I_{K} & 0-300 \mathrm{mV} \\ d_{c} & 0-30 \mathrm{~ms} \\ d_{c 1,} d_{c 2} & 0-30 \mathrm{~ms}, 1 \mathrm{~ms}\end{array}$

Control delay, direct control $d_{c 1,} d_{c 2} \quad 0-30 \mathrm{~ms}, 1 \mathrm{~ms}$

Excitatory-Inhibitory network

Control gain

$$
\begin{array}{cc}
K & 0-300 \mathrm{mV} \\
d_{c} & 0-30 \mathrm{~ms}
\end{array}
$$

Control delay, differential control $\quad d_{c 1}, d_{c 2} \quad 0-30 \mathrm{~ms}, 1 \mathrm{~ms}$ 